\title{
Antiliver Fibrosis Screening of Active Ingredients from Apium graveolens L. Seeds via GC-TOF-MS and UHPLC-MS/MS
}

\author{
Ming Qiao $\mathbb{D}^{1},{ }^{1}$ Jianhua Yang, ${ }^{2}$ Yao Zhao, ${ }^{1}$ Yi Zhu, ${ }^{2}$ Xiaomei Wang $\mathbb{D},{ }^{1}$ Xinling Wang, \\ and Junping $\mathrm{Hu} \mathbb{D i D}^{1}$ \\ ${ }^{1}$ College of Pharmacy, Xinjiang Medical University, Urumqi 830011, China \\ ${ }^{2}$ Department of Pharmacy, The First Affiliated Hospital, Xinjiang Medical University, Urumqi 830011, China
}

Correspondence should be addressed to Junping Hu; hjp-yft@163.com

Received 15 November 2019; Revised 13 January 2020; Accepted 27 January 2020; Published 19 February 2020

Academic Editor: Michel M. Machado

Copyright (c) 2020 Ming Qiao et al. This is an open access article distributed under the Creative Commons Attribution License, which permits unrestricted use, distribution, and reproduction in any medium, provided the original work is properly cited.

\begin{abstract}
Although several studies have been performed on Apium graveolens L.(celery) seeds, their antiliver fibrosis effects remain to be unexplored. Firstly, we detected the effects of celery seeds extracted with different concentrations of aqueous ethanol on the proliferation of HSC-LX2 cells. Then, we detected the effects of fractions of the optimal effect extract on the proliferation and apoptosis of HSC-LX2 cells. Finally, the compounds of petroleum ether (PP), ethyl acetate (PE), n-butyl alcohol (PB), and water fractions (PW) of the optimal effect extract were determined by GC-TOF-MS and UHPLC-MS/MS, to confirm the potentially antifibrotic compounds combined with pharmacodynamic experiment of monomer compounds in vitro. The results revealed that $60 \%$ ethanol extract of celery seeds (60-extract) exhibited remarkable inhibition effect on the proliferation of HSC-LX2 cells compared with $95 \%$ ethanol and aqueous extract. Besides, it validated that the inhibition rates of PP, $\mathrm{PE}, \mathrm{PB}$, and $\mathrm{PW}$ on the proliferation of HSC-LX2 cells were $75.14 \%, 73.52 \%, 54.09 \%$, and $43.36 \%$, and their percentage of apoptotic cells were $37.5 \%$, $4.3 \%, 0.7 \%$, and $0.1 \%$ at high doses, respectively. Additionally, it was manifested that apigenin, aesculetin, and butylphthalide have major contribution to the overall compounds of celery seeds, and the inhibition effects on the cell proliferation clearly elevated with increase in their contents. In essence, apigenin, aesculetin, and butylphthalide may hopefully become the natural products of antiliver fibrosis, which laid a foundation for the subsequent development of celery seeds as antiliver fibrosis drugs.
\end{abstract}

\section{Introduction}

Liver fibrosis is the essential pathophysiologic consequence of chronic hepatic injury [1]. Without favorable treatment, liver fibrosis can develop into cirrhosis, which is estimated to affect $1 \%$ to $2 \%$ of global population and result in over 1 million deaths annually worldwide $[2,3]$. The major causes of liver fibrosis include chronic hepatitis virus infection, alcohol abuse, and nonalcoholic steatohepatitis [4]. Hepatic fibrosis is characterized by excessive accumulation of extracellular matrix (ECM) caused by both increased synthesis and deposition of newly formed components and decreased or unbalanced degradation of ECM [5]. Hepatic stellate cell (HSC) activation is considered as a pivotal event in liver fibrosis, which is mainly responsible for the excessive accumulation of ECM proteins in the liver [6,7]. Nowadays, a growing body of basic and clinical evidences have manifested that liver fibrosis can be reversed after the cessation of injurious stimulus $[8,9]$. Hence, the development of antiliver fibrosis drugs is an urgent demand at present.

Apium graveolens L.(celery) belongs to the family Apiaceae, which is grown as a vegetable in many parts of the world [10]. It is rich in compounds including limonene, selinene, glycosides, flavonoids, and vitamins [11]. These constituents are also reported to prevent cardiovascular diseases [12], jaundice [13], urinary tract obstruction [14], gout [15], and rheumatic disorders [16, 17]. Celery seeds are spicy, carminative [18], diuretic [19], appetizer [20], stimulant, hypotensive [21], aphrodisiac, anti-inflammatory, and laxative [22]. In addition, celery seeds are also widely used in Chinese compound preparations to protect the liver, such as Huganbuzure Granule, Ganbaokang Granule, Fufangzupa Syrup, and Mawuliwusuli Granule [23, 24]. However, there is no report for pharmacological activity of celery seeds on 
the antiliver fibrosis. Therefore, transforming growth factor$\beta 1$ (TGF- $\beta 1$ ) induced HSC-LX2 cells to establish a liver fibrosis model in vitro to investigate the antiliver fibrosis effect of active fractions and compounds of celery seeds combined with GC-TOF-MS and UHPLC-MS/MS techniques.

\section{Materials and Methods}

2.1. Materials. Apium graveolens L. seeds were purchased from the decoction factory of Xinjiang Madison Medicine (Xinjiang, China). The botanical identification of the plant material was performed by Dr. Jianhua Yang from the first affiliated hospital of Xinjiang Medical University. The voucher specimen was deposited at the department of pharmacognosy of Xinjiang Medical University. Methanol, acetonitrile, and formic acid (HPLC grade) were obtained from Merck (Darmstadt, Germany). L-2-chlorophenylalanine and BSTFA (HPLC grade) were supplied by Sigma (St. Louis, MO, USA). Ultrapure water was prepared with a Milli-Q system (Millipore, Milford, MA, USA). Ethyl alcohol, petroleum ether, ethyl acetate, and n-butyl alcohol were of analytical grade.

2.1.1. Preparation of the Extracts. The celery seed extracts were prepared according to the reflux extraction, by adding 8 times the amount of distilled water, $60 \%$ and $95 \%$ aqueous ethanol (v/v) to $50.00 \mathrm{~g}$ of the dried celery seeds, respectively, and heating reflux 3 times $(3 \times 1.5 \mathrm{~h})$ at $60^{\circ} \mathrm{C}$. The filtrates were combined and concentrated with a rotary evaporator (IKA, Staufen, Germany) to obtain aqueous extract, $60 \%$ and $95 \%$ ethanol extracts, and the yields were $8.6 \%, 3.8 \%$ and $12.8 \%$, respectively.

\subsubsection{Preparation of the Fractions and Monomeric Com-} pounds from Celery Seeds. Celery seeds $(8.00 \mathrm{~kg})$ were refluxed thrice with 8 times the amount of $60 \%$ ethanol at $60^{\circ} \mathrm{C}$, and the extracts were combined and evaporated under reduced pressure to afford $200.6 \mathrm{~g}$ of crude extract. The $60 \%$ ethanol extract was dissolved in water to produce an aqueous solution, and then partitioned in turn with petroleum ether, ethyl acetate, and n-butyl alcohol to afford petroleum ether (PP, $5.12 \mathrm{~g}$ ), ethyl acetate (PE, $20.80 \mathrm{~g})$, n-butyl alcohol (PB, $70.60 \mathrm{~g}$ ), and water-soluble (PW, $89.60 \mathrm{~g}$ ) fractions after drying. Nine compounds were isolated from PP, PE, PB, and PW. Their structures were identified as apigenin $(8.3 \mathrm{mg})$ [25], 5-methoxypsoralen (10.2 mg) [26], apiin (9.1 mg) [27], rutin (7.7 mg) [28], kaempferol (9.3 mg) [29], luteolin (6.1 mg) [30], quercetin (6.2 mg) [31], mollugin (5.6 mg) [32], and butylphthalide ( $8.0 \mathrm{mg}$ ) [33] by using extensive spectroscopic analysis including ${ }^{1} \mathrm{H}-\mathrm{NMR}$ and ${ }^{13} \mathrm{C}-\mathrm{NMR}$.

2.1.3. Samples for GC-TOF-MS and UHPLC-MS/MS Analysis. $100 \mu \mathrm{L}$ of PP was extracted with methanol and added $10 \mu \mathrm{L}$ of L-2-chlorophenylalanine as internal standard and vortex (Grant, Cambridge, UK) mixed for 30 seconds. Then it was centrifuged at $4^{\circ} \mathrm{C}$ for $15 \mathrm{~min}$ at $12000 \mathrm{rpm}$ using a centrifuge (Thermo Fisher, Waltham, USA). $100 \mu \mathrm{L}$ of supernatant was transfered and dried completely in a vacuum concentrator (Retsch, Arzberg, Germany). To add $60 \mu \mathrm{L}$ of the BSTFA reagent to the sample aliquots, it is incubated for 1.5 hours at $70^{\circ} \mathrm{C}$. The sample was analysed by GC-TOF-MS. The PE, PB, and PW were thawed on the ice. Then the samples were centrifuged at $12000 \mathrm{rpm}$ for $15 \mathrm{~min}$ at $4^{\circ} \mathrm{C}$, and $200 \mu \mathrm{L}$ of supernatant of the sample was dried under a gentle nitrogen flow. The residue was reconstituted with $200 \mu \mathrm{L}$ of methanol. Then the samples were centrifuged at $12000 \mathrm{rpm}$ for 10 minutes at $4^{\circ} \mathrm{C}$. The resulting supernatants were transferred to $2 \mathrm{~mL}$ of sample vials and stored at $-80^{\circ} \mathrm{C}$ until the UHPLC-MS/MS analysis.

2.2. Cell Culture. HSC-LX2 cells (Applied Biosystems, Foster City, USA) were cultured in a high-glucose Dulbecco's modifed eagle medium (HG-DMEM, Gibco, Grand Island, USA) supplemented with 10\% FBS (Gibco, Grand Island, USA), $100 \mu \mathrm{g} \cdot \mathrm{mL}^{-1}$ streptomycin, and $100 \mathrm{U} \cdot \mathrm{mL}^{-1}$ penicillin (Gibco, Grand Island, USA). The cells were maintained in a humidified incubator with $5 \% \mathrm{CO}_{2}$ at $37^{\circ} \mathrm{C}$, and they were subcultured every 2 days to maintain logarithmic growth.

2.3. Establishment of the Liver Fibrosis Model Induced by TGF$\beta 1$. The logarithmic phase cells were inoculated in a 96-well plate at a density of $2 \times 10^{3}$ cells per well and treated with TGF- $\beta 1$ (Novoprotein, Shanghai, China) at concentrations of $5,10,20,40,80$, and $100 \mathrm{ng} \cdot \mathrm{mL}^{-1}$ for 24,48 , and $72 \mathrm{~h}$. Blank group (containing all reagents except the studied sample) was set. The cell viability was assessed by cellcounting kit-8 (CCK-8) assay (Solarbio, Beijing, China). The absorbance was determined at $450 \mathrm{~nm}$ using multiscan spectrum (Multiskan GO, Thermo Fisher, Waltham, USA). The hyaluronic acid (HA), laminin (LN), and type III procollagen (PCIII) levels were determined using enzymelinked immunosorbent assay (ELISA) kits (Jianglai Biological, Shanghai, China). All conditions were performed in triplicate, and each experiment repeated for three times. Cell viability was calculated as follows:

$$
\text { Cell viability }(\%)=\left[\frac{\left(\mathrm{OD}_{450 \text { (TGF- } \beta 1)}-\mathrm{OD}_{450 \text { (solvent) }}\right)}{\left(\mathrm{OD}_{450 \text { (blank) }}-\mathrm{OD}_{450 \text { (solvent) }}\right)}\right] \times 100 \text {. }
$$

2.4. Cell Proliferation Assay. HSC-LX2 cells were plated on 96-well plates at a density of $5 \times 10^{3}$ cells per well and treated with various concentrations of extracts and fractions of celery seed in combination with $100 \mathrm{ng} \cdot \mathrm{mL}^{-1}$ of TGF- $\beta 1$ for $48 \mathrm{~h}$. Blank group (containing all reagents except the studied sample) was set. The positive control group was added with $50 \mu \mathrm{g} \cdot \mathrm{mL}^{-1}$ of the compound Biejiaruangan Troche (BJRG) (Inner Mongolia Furui Medical Science, Inner Mongolia, China). $100 \mathrm{ng} \cdot \mathrm{mL}^{-1}$ of TGF- $\beta 1$ was added to the model group. The absorbance was quantified at $450 \mathrm{~nm}$ using a multiscan spectrum. Additionally, the HA, LN, and PCIII 
levels were also performed by ELISA kits. Each treatment was performed in triplicate, and each experiment was repeated for three times. Cell inhibiting rate was calculated as follows:

$$
\text { cell inhibiting rate }(\%)=\left[1-\left[\frac{\left(\mathrm{OD}_{450 \text { (sample) }}-\mathrm{OD}_{450 \text { (solvent) }}\right)}{\left(\mathrm{OD}_{450(T G F-\beta 1)}-\mathrm{OD}_{450 \text { (solvent) }}\right)}\right]\right] \times 100
$$

2.5. Cell Apoptosis. Annexin V-FITC Apoptosis Detection Kit (Becton Dickinson, Franklin Lakes, NJ, USA) was utilized for cell apoptosis analysis according to the manufacturer's instruction. Cells were divided into viable cells, dead cells, early apoptotic cells and apoptotic cells, and analyses were performed on the flow cytometer (Becton Dickinson, Franklin Lakes, NJ, USA). The ratio of apoptotic cell reflected the proapoptotic effect of PP, PE, PB, and PW of $60 \%$ ethanol extract from celery seeds. Each treatment was repeated in triplicate.

2.6. GC-TOF-MS Analysis. The Agilent 7890 gas chromatograph system is coupled with a Pegasus HT time-offlight mass spectrometer. The system utilized a DB-5MS capillary column coated with 5\% diphenyl cross-linked with 95\% dimethylpolysiloxane $(30 \mathrm{~m} \times 250 \mu \mathrm{m}, 0.25 \mu \mathrm{m}$; J\&W Scientific, Folsom, CA, USA). An aliquot of the analyte $(1 \mu \mathrm{L})$ was injected into the GC/MS apparatus. Helium was the carrier gas at a flow rate of $1 \mathrm{~mL} \cdot \mathrm{min}^{-1}$. GC oven temperature started at $50^{\circ} \mathrm{C}$ and was held for $1 \mathrm{~min}$ at $310^{\circ} \mathrm{C}$ and then for $10 \mathrm{~min}$ with program rate $10^{\circ} \mathrm{C} \cdot \mathrm{min}^{-1}$. The injector and detector temperatures were set at $280^{\circ} \mathrm{C}$ and $250^{\circ} \mathrm{C}$, respectively. The mass spectrometer was run in the electron ionization mode $(-70 \mathrm{eV})$.

2.7. UHPLC-MS/MS Analysis. Chromatographic analysis was performed in an Agilent 1290 ultra-high performance liquid chromatography (Agilent, Technologies, CA, USA). An ACQUITY ULC HSS T3 column $(2.1 \mathrm{~mm} \times 100 \mathrm{~mm}$, $1.8 \mu \mathrm{m}$, Waters, USA) was used for separation with gradient elution of $0.1 \%$ formic acid aqueous solution (A) and acetonitrile (B), and the column temperature was maintained at $40^{\circ} \mathrm{C}$. The detailed gradient conditions were 0-2 minutes, $0 \%-2 \% \mathrm{~B}$; 2-11 minutes, $2 \%-98 \% \mathrm{~B}$; and $11-13$ minutes, $98 \%-2 \% \mathrm{~B}$. The flow rate was $0.3 \mathrm{~mL} \cdot \mathrm{min}^{-1}$, and the injection volume was $10 \mu \mathrm{L}$. Mass spectrometry was performed on the AB Sciex QTrap 6500 (AB SCIEX, Los Angeles, CA, USA) for characterization equipped with electrospray ionization (ESI) source in both positive and negative ionization multiple reaction monitoring (MRM) mode. The parameters were set as follows: nebulizer gas of 60 psi; heater gas of 55 psi; curtain gas of 35 psi; ionspray voltage of $+5500 /-4500 \mathrm{~V}$; ion transfer tube temperature of $550^{\circ} \mathrm{C}$.

2.8. Screening of Antiliver Fibrosis Activity of Monomeric Compounds. Briefly, cells were seeded onto a 96-well plate at a density of $5 \times 10^{3}$ cells per well. Cells were exposed to different concentrations of compounds with $100 \mathrm{ng} \cdot \mathrm{mL}^{-1}$ TGF- $\beta 124 \mathrm{~h}$ after seeding. The positive control group was added with $50 \mu \mathrm{g} \cdot \mathrm{mL}^{-1}$ of BJRG. Each treatment was repeated in triplicate. After $48 \mathrm{~h}$ of sample incubation at $37^{\circ} \mathrm{C}$, CCK- 8 solution was added to each well. After a further $1 \mathrm{~h}$ of incubation, the absorbance of each well was measured at $450 \mathrm{~nm}$, and cell inhibiting rate was calculated.

2.9. Statistical Analysis. The results of cell experiment were expressed as the mean standard deviation of three parallel measurements, and statistical significance was assessed using one-way analysis of variance (ANOVA) followed by a multiple comparison test (Tukey's post-hoc test), where $P<0.05$ and $P<0.01$ were considered significant. Three injections used in the GC-TOF-MS and UHPLC-MS/MS analysis, and the relative percentage of the compound was the average of the normalized values of the three determinations of the peak area.

\section{Results}

3.1. Effects of TGF- $\beta 1$ on the Proliferation of HSC-LX2 Cells. We used six concentrations and three time points of TGF- $\beta 1$ to explore the proliferation of HSC-LX2 cells (Table 1). At given time points, HSC-LX2 cells viability clearly elevated with increased TGF- $\beta 1$ concentrations. The HSC-LX 2 cells viability remarkably decreased with prolonged time. Absorbance peaked at $48 \mathrm{~h}$ with $100 \mathrm{ng} \cdot \mathrm{mL}^{-1}$ of TGF- $\beta 1$ and abated gradually later. Therefore, HSC-LX2 cells were activated by $100 \mathrm{ng} \cdot \mathrm{mL}^{-1}$ of TGF- $\beta 1$ for $48 \mathrm{~h}$ to establish a liver fibrosis model.

3.2. Effects of TGF- $\beta 1$ on Liver Fibrosis Indexes. As expected, serum HA, LN, and PCIII levels were remarkably elevated after TGF- $\beta 1$ treatment in comparison with the blank control group. It indicated that the liver fibrosis model was established successfully in vitro (Table 2).

3.3. Effects of Extracts and the Fractions of Celery Seeds on Proliferation of HSC-LX2 Cells. HSC-LX2 cells were notably activated by TGF- $\beta 1$, as shown in Table 3 . The results indicated that celery seed extracts and its fractions inhibited HSC-LX2 cell proliferation in a dose-dependent fashion. $60 \%$ ethanol extract showed a stronger inhibitory effect than the aqueous extract and 95\% ethanol extract. The inhibition rates of $\mathrm{PP}, \mathrm{PE}, \mathrm{PB}$, and $\mathrm{PW}$ were 75.14\%, 73.52\%, 54.09\%, and $43.36 \%$ at high doses, respectively.

3.4. Effects of PP, PE, $P B$, and $P W$ on Liver Fibrosis Indexes. TGF- $\beta 1$ treatment caused a remarkable collagen accumulation. In sharp contrast, treatment with $\mathrm{PW}, \mathrm{PB}, \mathrm{PE}$, and $\mathrm{PP}$ 
TABLE 1: Effects of TGF- $\beta 1$ on the viability of HSC-LX2 cells (mean \pm SD, $n=3$ ).

\begin{tabular}{lccccccc}
\hline \multirow{2}{*}{ Group } & \multirow{2}{*}{ Dose $\left(\mathrm{ng} \cdot \mathrm{mL}^{-1}\right)$} & \multicolumn{2}{c}{$24 \mathrm{~h}$} & \multicolumn{2}{c}{$48 \mathrm{~h}$} & \multicolumn{2}{c}{$72 \mathrm{~h}$} \\
& & OD & Viability (\%) & OD & Viability (\%) & OD & Viability (\%) \\
\hline Solvent control & - & $0.183 \pm 0.004$ & - & $0.175 \pm 0.004$ & - & $0.210 \pm 0.001$ \\
\hline Blank control & - & $0.395 \pm 0.102$ & - & $0.671 \pm 0.023$ & - & $1.739 \pm 0.197$ & - \\
\hline & 5 & $0.347 \pm 0.054$ & $77.35 \pm 0.75$ & $0.711 \pm 0.072$ & $108.11 \pm 3.26$ & $0.573 \pm 0.109^{\#}$ & $23.76 \pm 2.65$ \\
& 10 & $0.501 \pm 0.028$ & $150.72 \pm 0.62$ & $0.775 \pm 0.042$ & $121.10 \pm 2.61$ & $0.596 \pm 0.035^{\#}$ & $25.24 \pm 2.41$ \\
TGF- $\beta 1$ & 20 & $0.511 \pm 0.067$ & $154.71 \pm 0.86$ & $0.820 \pm 0.103$ & $130.12 \pm 4.51$ & $1.316 \pm 0.136$ & $47.63 \pm 1.04$ \\
& 40 & $0.695 \pm 0.105^{\#}$ & $215.52 \pm 1.96$ & $0.894 \pm 0.110$ & $145.21 \pm 5.62$ & $1.311 \pm 0.185$ & $72.13 \pm 3.65$ \\
& 80 & $0.640 \pm 0.108^{\#}$ & $241.49 \pm 0.21$ & $1.093 \pm 0.079^{\#}$ & $177.59 \pm 2.54$ & $2.472 \pm 0.145^{\#}$ & $148.51 \pm 2.54$ \\
& 100 & $0.803 \pm 0.101^{\#}$ & $292.45 \pm 0.26$ & $1.755 \pm 0.081^{\# \#}$ & $318.39 \pm 6.26$ & $2.728 \pm 0.177^{\#}$ & $164.54 \pm 3.22$ \\
\hline
\end{tabular}

Compared with the blank control group $\left({ }^{\#} P<0.05,{ }^{\# \#} P<0.01\right)$.

TABLE 2: Effects of TGF- $\beta 1$ on liver fibrosis indexes (mean $\pm \mathrm{SD}, n=3$ ).

\begin{tabular}{|c|c|c|c|c|}
\hline Group & Dose $\left(\mathrm{ng} \cdot \mathrm{mL}^{-1}\right)$ & $\mathrm{LN}\left(\mu \mathrm{g} \cdot \mathrm{L}^{-1}\right)$ & HA (ng. $\left.L^{-1}\right)$ & PCIII $\left(\mu \mathrm{g} \cdot \mathrm{L}^{-1}\right)$ \\
\hline Blank control & - & $51.8 \pm 0.78$ & $26.9 \pm 5.67$ & $18.8 \pm 4.53$ \\
\hline Model group (TGF- $\beta 1$ ) & 100 & $833.4 \pm 9.94^{\# \#}$ & $302.3 \pm 7.44^{\# \#}$ & $115.3 \pm 3.38^{\# \#}$ \\
\hline
\end{tabular}

TABLE 3: Effects of the extracts and fractions of celery seeds on inhibition of HSC-LX2 cell proliferation (mean \pm SD, $n=3$ ).

\begin{tabular}{|c|c|c|c|c|}
\hline Group & Fraction & Dose $\left(\mu \mathrm{g} \cdot \mathrm{mL}^{-1}\right)$ & OD & Inhibition ratio (\%) \\
\hline Solvent control & & - & $0.254 \pm 0.019$ & - \\
\hline Blank control & & 0 & $1.021 \pm 0.079$ & - \\
\hline Model group (TGF- $\beta 1$ ) & & 0.1 & $3.522 \pm 0.178^{\# \#}$ & - \\
\hline BJRG & & 50 & $1.116 \pm 0.012^{* *}$ & $73.11 \pm 0.87$ \\
\hline \multirow{3}{*}{ Aqueous extract of celery seeds } & & 50 & $3.532 \pm 0.227^{\# \#}$ & - \\
\hline & & 100 & $3.074 \pm 0.131^{* \# \#}$ & $13.79 \pm 2.91$ \\
\hline & & 200 & $2.537 \pm 0.541^{* \#}$ & $30.13 \pm 1.02$ \\
\hline \multirow{3}{*}{$95 \%$ ethanol extract of celery seeds } & & 50 & $3.602 \pm 0.281$ & - \\
\hline & & 100 & $2.926 \pm 0.0526^{* \#}$ & $18.22 \pm 1.69$ \\
\hline & & 200 & $1.717 \pm 0.026^{* * \#}$ & $55.22 \pm 2.44$ \\
\hline \multirow{15}{*}{$60 \%$ ethanol extract of celery seeds } & \multirow{7}{*}{$\mathrm{PP}$} & 50 & $2.123 \pm 0.138^{* * \#}$ & $42.75 \pm 2.21$ \\
\hline & & 100 & $1.351 \pm 0.052^{* *}$ & $66.42 \pm 1.53$ \\
\hline & & 200 & $1.097 \pm 0.022^{* *}$ & $74.20 \pm 2.38$ \\
\hline & & 25 & $2.598 \pm 0.224^{* \#}$ & $28.26 \pm 1.29$ \\
\hline & & 50 & $1.619 \pm 0.129^{* * \#}$ & $58.21 \pm 2.11$ \\
\hline & & 75 & $1.066 \pm 0.023^{* *}$ & $75.14 \pm 1.45$ \\
\hline & & 25 & $2.893 \pm 0.159^{* \#}$ & $19.23 \pm 1.32$ \\
\hline & \multirow[t]{3}{*}{$\mathrm{PE}$} & 50 & $2.001 \pm 0.239^{* * \#}$ & $46.55 \pm 3.87$ \\
\hline & & 75 & $1.119 \pm 0.312^{* *}$ & $73.52 \pm 2.36$ \\
\hline & & 25 & $2.956 \pm 0.239^{* \#}$ & $17.30 \pm 3.13$ \\
\hline & \multirow[t]{3}{*}{$\mathrm{PB}$} & 50 & $1.916 \pm 0.209^{* * \#}$ & $49.24 \pm 2.03$ \\
\hline & & 75 & $1.754 \pm 0.241^{* * \#}$ & $54.09 \pm 1.44$ \\
\hline & & 25 & $3.369 \pm 0.319^{\# \#}$ & $4.68 \pm 0.75$ \\
\hline & \multirow[t]{2}{*}{$\mathrm{PW}$} & 50 & $2.889 \pm 0.162^{* \#}$ & $19.36 \pm 1.72$ \\
\hline & & 75 & $2.105 \pm 0.311^{* * \#}$ & $43.36 \pm 3.55$ \\
\hline
\end{tabular}

Compared with the blank control group $\left({ }^{\#} P<0.05,{ }^{\#} P<0.01\right)$; compared with the model group $\left({ }^{*} P<0.05,{ }^{* *} P<0.01\right)$.

markedly decreased hepatic collagen matrix accumulation, as shown in Table 4. The serum LN, HA, and PCIII contents were markedly reduced in the PE and PP groups, and that of $\mathrm{PP}$ groups were closer to the control groups. PW, PB, PE, and PP exhibited different degree in antiliver fibrosis, which might be related to the active component contents.
3.5. Apoptosis Analysis. Annexin V-FITC/PI double staining and flow cytometry were performed to compare the apoptotic rates of HSC-LX2 cells in different groups. As shown in Figure 1, the percentage of apoptotic cells was $0.1 \%$ in the control group (Figure 1(a)), that of the group treated with BJRG, PP, PE, PB, and PW were 2.6\%, 37.5\%, 4.3\%, 0.7\%, 
TABLe 4: Effects of PP, PE, PB, and PW on liver fibrosis indexes (mean $\pm \mathrm{SD}, n=3$ ).

\begin{tabular}{|c|c|c|c|c|}
\hline Group & Dose $\left(\mu \mathrm{g} \cdot \mathrm{mL}^{-1}\right)$ & $\mathrm{LN}\left(\mu \mathrm{g} \cdot \mathrm{L}^{-1}\right)$ & HA $\left(\right.$ ng $\left.\cdot \mathrm{L}^{-1}\right)$ & PCIII $\left(\mu \mathrm{g} \cdot \mathrm{L}^{-1}\right)$ \\
\hline Blank control & 0 & $51.8 \pm 0.78$ & $26.9 \pm 5.67$ & $18.8 \pm 4.53$ \\
\hline BJRG & 50 & $211.0 \pm 5.08^{* *}$ & $64.5 \pm 3.19^{*}$ & $29.2 \pm 2.53^{* *}$ \\
\hline Model group (TGF- $\beta 1$ ) & 0.1 & $833.4 \pm 9.94^{\# \#}$ & $302.3 \pm 7.44^{\# \#}$ & $115.3 \pm 3.38^{\# \#}$ \\
\hline PW & 75 & $638.27 \pm 7.22^{\# \Delta \Delta}$ & $178.82 \pm 3.94^{\# * \Delta}$ & $108.87 \pm 9.29^{\# \# \Delta \Delta}$ \\
\hline $\mathrm{PB}$ & 75 & $502.56 \pm 5.85^{\# * \Delta \Delta}$ & $111.37 \pm 4.95^{\# * \Delta}$ & $78.36 \pm 5.22^{\# \Delta}$ \\
\hline $\mathrm{PE}$ & 75 & $118.40 \pm 5.74^{* *}$ & $70.80 \pm 7.07^{*}$ & $28.30 \pm 0.21^{* *}$ \\
\hline $\mathrm{PP}$ & 75 & $97.32 \pm 6.30^{* *}$ & $30.91 \pm 5.22^{* *}$ & $19.01 \pm 4.26^{* *}$ \\
\hline
\end{tabular}

Compared with the blank control group $\left({ }^{\#} P<0.05,{ }^{\#} P<0.01\right)$; compared with the model group $\left({ }^{*} P<0.05,{ }^{* *} P<0.01\right)$; compared with the PP group, $\left({ }^{\Delta} P<0.05,{ }^{\Delta \Delta} P<0.01\right)$.

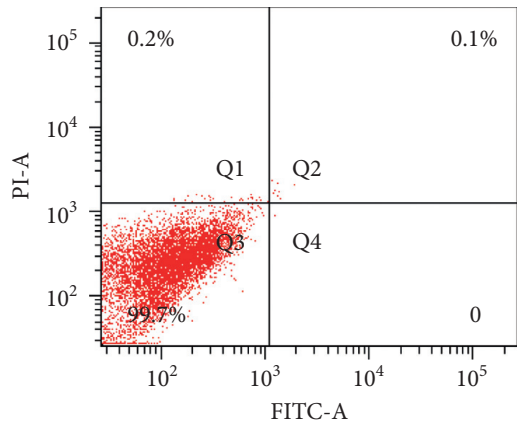

(a)

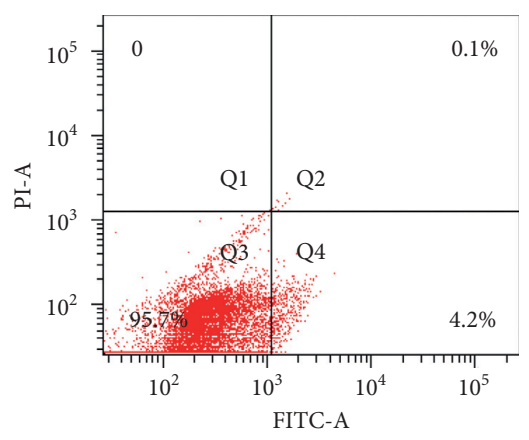

(d)

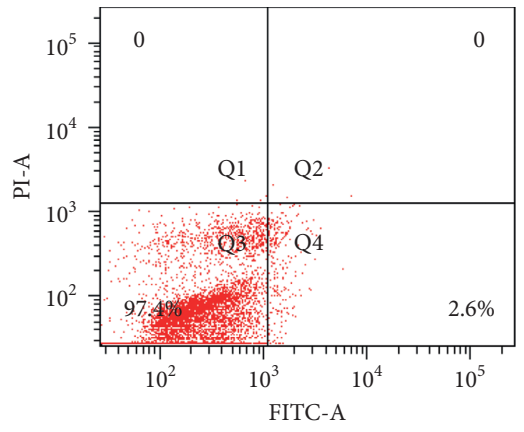

(b)

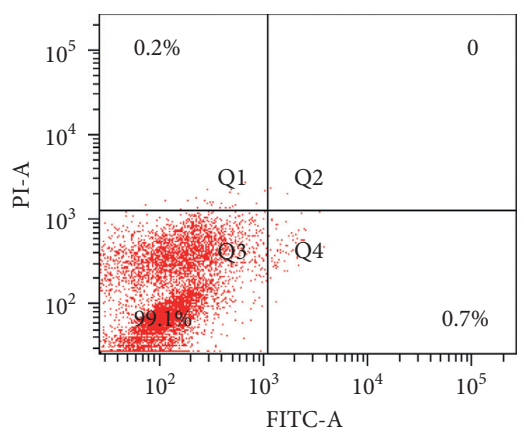

(e)

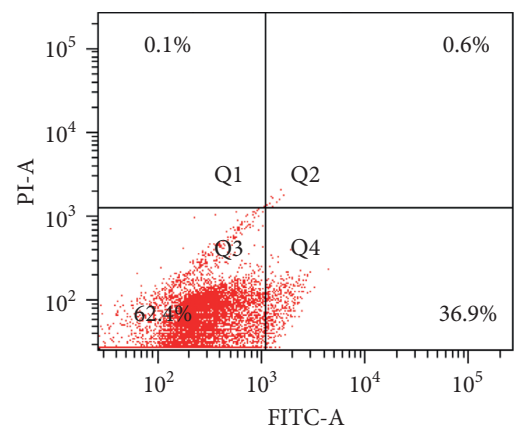

(c)

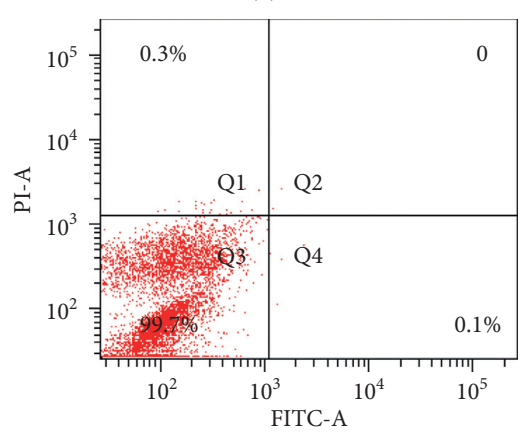

(f)

Figure 1: Effects of PP, PE, PB, and PW on apoptosis of HSC-LX2 cells. (a) Control. (b) BJRG. (c) PP. (d) PE. (e) PB. (f) PW.

and $0.1 \%$, respectively (Figures $1(\mathrm{~b})-1(\mathrm{f})$ ). PP showed stronger proapoptotic function than that of the positive control.

3.6. Analysis of the Fractions of Celery Seeds Using GC-TOFMS and UHPLC-MS/MS Methods. Identification of several compounds was enabled by comparison of retention times, MS spectra, and MS/MS spectral data with the standard substance and reference materials reported in the literature. According to our knowledge, the chemical compositions of the fractions from celery seeds, investigated by GC-TOF-MS and UHPLC-MS/MS, was performed for the first time in this study. 122 chemical components were identified from PP, $\mathrm{PE}, \mathrm{PB}$, and PW through analysis of GC-TOF-MS and UHPLC-MS/MS (Table 5). The obtained total ion chromatograms are shown in Figure 2. The 378 peaks of $P P$ were separated in GC and preliminarily identified 39 main compounds belonging to different chemical families by
TOF-MS which account for $94.32 \%$ of total peak area. Chemical composition analysis showed that phenyl peptides accounted for $24.56 \%$ acids, $22.96 \%$ aldehydes $19.37 \%$, flavonoids $18.42 \%$, alcohols $3.24 \%$, and major compounds were farnesal 19.37\%, butylphthalide 18.42\%, 4-hydroxymethyl 3-methoxyphenoxyacetic acid 13.54\%, aesculetin $13.28 \%$, and apigenin $11.28 \%$.

Of all the compounds detected by UHPLC-MS/MS, 90 compounds were identified by searching the Biotree DB Database and comparing their retention times and MS spectra with the reference literature. 52 chemical constituents were identified from PE, mainly flavonoids, amides, pigments, and ketones, accounting for $75.86 \%$ of the total peak area. The major constituents that have been determined through UHPLC-MS/MS analyses were apigenin 8.48\%, 2acetyl-1-ethylpyrrole $5.22 \%$, aesculetin $4.32 \%$, and 2 -phenylacetamide $3.86 \%$. 48 compounds were identified from $\mathrm{PB}$, mainly glycosides, esters, aldehydes, and ketones, accounting for $81.88 \%$ of the total peak area. The main 
TABLE 5: Identification of chemical compositions of fractions from celery seeds.

\begin{tabular}{|c|c|c|c|c|c|c|}
\hline \multirow{2}{*}{ No. } & \multirow{2}{*}{$\mathrm{RT}^{\mathrm{a}}(\min )$} & \multirow{2}{*}{ Compound } & \multicolumn{4}{|c|}{ Peak Area $^{\mathrm{b}}(\%)$} \\
\hline & & & PW & $\mathrm{PB}$ & $\mathrm{PE}$ & $\mathrm{PP}$ \\
\hline 1 & 0.69 & 6-Hydroxyangelicin & 0.40 & - & - & - \\
\hline 2 & 0.89 & Mannitol & - & 0.43 & - & - \\
\hline 3 & 0.91 & 3-Buten-2-one 1-(2,3,6-trimethyl phenyl) & 1.37 & 0.23 & - & - \\
\hline 4 & 0.96 & Lactulose & - & 0.28 & - & - \\
\hline 5 & 0.97 & Ectoine & - & 1.81 & - & - \\
\hline 6 & 0.98 & Heptyl formate & 0.45 & 0.38 & - & - \\
\hline 7 & 0.99 & Genipic acid & 0.45 & - & - & - \\
\hline 8 & 1.00 & 2,4-Diethylthiazole & - & 1.56 & - & - \\
\hline 9 & 1.05 & 2,5-Dioxopentanoate & 2.18 & - & - & - \\
\hline 10 & 1.24 & 2-Hydroxyethanesulfonate & 0.40 & 0.39 & - & - \\
\hline 11 & 1.27 & 2,6-Piperidinedicarboxylic acid & 1.09 & - & - & - \\
\hline 12 & 1.37 & Pregabalin & 1.99 & - & - & - \\
\hline 13 & 1.38 & $\mathrm{~N}$-acetylhistamine & 1.46 & - & - & - \\
\hline 14 & 1.38 & Nipecotic acid & 11.99 & 1.51 & - & - \\
\hline 15 & 1.39 & 4-Guanidinobutanoic acid & - & 0.25 & - & - \\
\hline 16 & 1.44 & Gln gly & 1.87 & - & - & - \\
\hline 17 & 1.52 & Cytarabine & - & 0.74 & - & - \\
\hline 18 & 1.62 & Ser val gly & - & 2.46 & - & - \\
\hline 19 & 1.74 & Ormetoprim & 0.29 & - & - & - \\
\hline 20 & 2.02 & 6-Hydroxynicotinic acid & - & - & 0.33 & - \\
\hline 21 & 2.54 & 3,3,5-Triiodo-L-thyronine-beta-D-glucuronoside & - & 0.35 & - & - \\
\hline 22 & 3.13 & 2-Phenylacetamide & 0.63 & 0.52 & 3.86 & 0.10 \\
\hline 23 & 4.1 & (S)-5'-deoxy-5'-(methylsulfinyl)adenosine & - & 0.54 & - & - \\
\hline 24 & 4.12 & Adenine & - & 1.88 & - & - \\
\hline 25 & 4.12 & Adenosine & 0.39 & 14.99 & 0.47 & - \\
\hline 26 & 4.19 & Cordycepin & - & 0.32 & - & - \\
\hline 27 & 4.31 & Patulin & 0.42 & - & - & - \\
\hline 28 & 4.34 & 4-Pyridoxic acid & - & 0.27 & 0.36 & - \\
\hline 29 & 4.35 & Inosine & - & 0.94 & - & - \\
\hline 30 & 4.36 & $3^{\prime}$-Deoxyadenosine & - & - & 0.41 & - \\
\hline 31 & 4.39 & $\alpha$-[1-(ethylamino)ethyl]-p-hydroxy- benzyl alcohol & - & - & 0.56 & - \\
\hline 32 & 4.93 & Selfotel & 1.51 & - & - & - \\
\hline 33 & 4.98 & 2-Acetyl-1-ethylpyrrole & 3.72 & 0.30 & 5.22 & 0.08 \\
\hline 34 & 4.99 & 3-Aminobenzamide & 0.87 & - & 0.93 & - \\
\hline 35 & 5.01 & 4-Hydroxydihydrocinnamaldehyde & - & - & 0.36 & - \\
\hline 36 & 5.11 & Imidazoleacetic acid & - & - & 0.79 & - \\
\hline 37 & 5.16 & Mequinol & 0.34 & 2.55 & - & - \\
\hline 38 & 5.36 & 2-Pyrocatechuic acid & 0.62 & - & 0.85 & - \\
\hline 39 & 5.36 & L-tryptophan & 1.52 & 0.44 & - & - \\
\hline 40 & 5.39 & Imidacloprid-guanidine & 0.39 & 0.27 & 1.07 & - \\
\hline 41 & 5.50 & Dopamine & - & - & 0.50 & - \\
\hline 42 & 5.76 & Methiocarb-sulfoxide & 0.45 & - & - & - \\
\hline 43 & 5.81 & 3-(4-methoxy-6-oxopyran-2-yl)butanoic acid & 0.51 & - & - & - \\
\hline 44 & 6.24 & 1-naphthylmethanol & - & - & 0.89 & - \\
\hline 45 & 6.41 & 1H-indole-3-carboxaldehyde & 2.65 & 0.32 & 1.86 & 0.38 \\
\hline 46 & 6.73 & n-Acetylhomoproline & - & 0.23 & - & - \\
\hline 47 & 6.77 & Methyl 3-methyl-1-butenyl disulfide & 0.75 & 0.28 & - & - \\
\hline 48 & 6.82 & Aesculin & - & - & 0.66 & - \\
\hline 49 & 6.87 & Oleic acid & - & - & 0.47 & - \\
\hline 50 & 6.89 & Beta-carboline & - & - & 0.86 & - \\
\hline 51 & 6.98 & 3-Carboxypropyl trimethylammonium & 0.43 & - & 0.39 & - \\
\hline 52 & 6.99 & Apigenin & - & - & 8.48 & 11.28 \\
\hline 53 & 7.28 & trans-O-hydroxybenzylidenepyruvate & 0.35 & - & - & - \\
\hline 54 & 7.31 & Eugenitol & 0.85 & 0.22 & 0.84 & - \\
\hline 55 & 7.54 & Glycolic acid & - & - & - & 0.18 \\
\hline 56 & 7.59 & 2-Ketobutyric acid & - & - & - & 0.12 \\
\hline 57 & 7.61 & Indoleacetic acid & 0.44 & - & 0.43 & - \\
\hline 58 & 7.62 & 4-Methyl-1-phenyl-2-pentanone & 1.35 & - & - & - \\
\hline 59 & 7.66 & 3-Methyl-1-phenyl-3-pentanol & 8.05 & - & 0.72 & - \\
\hline
\end{tabular}


TABle 5: Continued.

\begin{tabular}{|c|c|c|c|c|c|c|}
\hline \multirow{2}{*}{ No. } & \multirow{2}{*}{$\mathrm{RT}^{\mathrm{a}}(\min )$} & \multirow{2}{*}{ Compound } & \multicolumn{4}{|c|}{ Peak Areab $^{\mathrm{b}}(\%)$} \\
\hline & & & PW & $\mathrm{PB}$ & $\mathrm{PE}$ & $\mathrm{PP}$ \\
\hline 60 & 7.69 & $3^{\prime}$-Methoxy-E,E-dienoestrol & 0.36 & - & 0.70 & - \\
\hline 61 & 7.79 & 2-Butyl-1-octanol & 0.38 & - & 0.52 & - \\
\hline 62 & 7.80 & 2-Hydroxy-8-methylchromene-2-carboxylate & 0.43 & - & 1.91 & - \\
\hline 63 & 7.84 & Diflufenican & - & 1.78 & 0.82 & - \\
\hline 64 & 7.89 & 3-Methylbenzaldehyde & 0.40 & - & 0.42 & - \\
\hline 65 & 7.98 & Luteolin 7 -galactoside & 6.59 & 2.42 & 0.76 & - \\
\hline 66 & 7.99 & 2-Naphthol & 2.30 & 0.24 & 0.94 & - \\
\hline 67 & 8.16 & Apiin & 2.34 & 3.64 & 0.78 & - \\
\hline 68 & 8.17 & Ambolin & - & 6.72 & 1.49 & - \\
\hline 69 & 8.19 & 2-Hydroxymuconate semialdehyde & 1.29 & 5.21 & 1.53 & - \\
\hline 70 & 8.20 & Decanoic acid, 3-amino-, (S)- & - & 0.66 & 2.77 & - \\
\hline 71 & 8.21 & Indoleacrylic acid & 0.54 & - & 1.82 & - \\
\hline 72 & 8.22 & Glycine & - & - & - & 0.11 \\
\hline 73 & 8.27 & Aesculetin & 0.11 & 0.56 & 4.32 & 13.28 \\
\hline 74 & 8.27 & Pelargonidin 3-O-glucoside & - & 2.08 & 2.61 & - \\
\hline 75 & 8.32 & Peonidin-3-O-beta-galactopyranoside & 0.18 & 1.95 & 2.97 & - \\
\hline 76 & 8.46 & 2-Butyl-3-phenyl-2-propen-1-al & 0.79 & 0.35 & 0.95 & - \\
\hline 77 & 8.61 & 1,6-Dimethoxypyrene & 0.30 & - & 2.08 & - \\
\hline 78 & 8.65 & Foliosidine & 0.51 & - & 1.76 & - \\
\hline 79 & 8.72 & Ensulizole & 2.23 & - & 1.53 & - \\
\hline 80 & 8.73 & 3-Hydroxypropionic acid & - & - & - & 2.61 \\
\hline 81 & 8.84 & Flavan skeleton & 0.40 & - & 1.82 & - \\
\hline 82 & 8.92 & Chalconaringenin & - & 4.87 & - & - \\
\hline 83 & 9.01 & Peonidin 3-(6" -acetylglucoside $)$ & - & - & 1.82 & - \\
\hline 84 & 9.27 & Mesylate & 7.13 & 3.03 & 0.59 & - \\
\hline 85 & 9.33 & 15-Methylpalmitate (2R,3R)-2-(3,4-dihydroxyphenyl)-3,5- & 0.12 & 7.18 & 2.26 & - \\
\hline 86 & 9.40 & Dihydroxy-7-methoxy-2,3-dihydrochromen-4-one & 1.86 & 1.17 & - & - \\
\hline 87 & 9.70 & $\alpha$-Terpinene & 0.45 & 0.51 & 0.37 & 0.47 \\
\hline 88 & 9.80 & 4-Methyl-N-ethylcathinone & 0.54 & 1.43 & 0.86 & - \\
\hline 89 & 9.93 & Perillyl acetate & 0.65 & 0.26 & 1.39 & - \\
\hline 90 & 10.27 & 5,6,7,8-Tetrahydro-2-naphthol & 1.12 & 2.36 & 1.49 & - \\
\hline 91 & 10.52 & Cicaprost & - & 0.25 & 2.47 & - \\
\hline 92 & 13.72 & Erucamide & 2.27 & 0.75 & 0.80 & 0.09 \\
\hline 93 & 13.91 & Oxoproline & - & - & - & 0.16 \\
\hline 94 & 14.52 & $(2 \mathrm{R}, 3 \mathrm{~S})-2$-Hydroxy-3-isopropylbutanedioic acid & - & - & - & 0.16 \\
\hline 95 & 15.44 & Vanillin & - & - & - & 0.39 \\
\hline 96 & 15.51 & Lauric acid & - & - & - & 0.17 \\
\hline 97 & 15.80 & DL-anabasine & - & - & - & 1.79 \\
\hline 98 & 15.93 & Phthalic acid & - & - & - & 0.35 \\
\hline 99 & 16.01 & Methyl jasmonate & - & - & - & 1.44 \\
\hline 100 & 16.23 & D-arabitol & - & - & - & 0.58 \\
\hline 101 & 16.24 & Ribitol & - & - & - & 0.42 \\
\hline 102 & 16.59 & $\mathrm{~N}$-(2-hydroxyethyl)-iminodiacetic acid & - & - & - & 0.10 \\
\hline 103 & 16.68 & Farnesal & - & - & - & 19.37 \\
\hline 104 & 16.72 & S-carboxymethylcysteine & - & - & - & 0.17 \\
\hline 105 & 16.87 & Gentisic acid & - & - & - & 1.81 \\
\hline 106 & 16.97 & terephthalic acid & - & - & - & 1.10 \\
\hline 107 & 17.07 & Farnesol & - & - & - & 0.40 \\
\hline 108 & 17.13 & $\mathrm{~N}$-acetylisatin & - & - & - & 0.16 \\
\hline 109 & 17.64 & Myristic acid & - & - & - & 0.47 \\
\hline 110 & 17.76 & Fructose & 0.78 & - & - & - \\
\hline 111 & 18.08 & Butylphthalide & - & - & - & 18.42 \\
\hline 112 & 18.09 & Galactose & 0.10 & - & - & - \\
\hline 113 & 18.59 & Coniferyl alcohol & - & - & - & 0.82 \\
\hline 114 & 18.70 & 1-Hexadecanol & - & - & - & 0.12 \\
\hline 115 & 19.45 & 4-Hydroxymethyl-3-methoxyphenoxyacetic acid & - & - & - & 13.54 \\
\hline 116 & 19.62 & Palmitic acid & - & - & - & 0.64 \\
\hline 117 & 19.94 & Myo-inositol & - & - & - & 0.08 \\
\hline 118 & 20.61 & 6-Hydroxy caproic acid dimer & - & - & - & 0.35 \\
\hline
\end{tabular}


TABle 5: Continued.

\begin{tabular}{|c|c|c|c|c|c|c|}
\hline \multirow{2}{*}{ No. } & \multirow{2}{*}{$\mathrm{RT}^{\mathrm{a}}(\min )$} & \multirow{2}{*}{ Compound } & \multicolumn{4}{|c|}{ Peak Area $^{\mathrm{b}}(\%)$} \\
\hline & & & PW & $\mathrm{PB}$ & $\mathrm{PE}$ & PP \\
\hline 119 & 21.17 & Linoleic acid & - & - & - & 0.78 \\
\hline 120 & 21.40 & Stearic acid & - & - & - & 0.76 \\
\hline 121 & 28.89 & Cholestan-3beta-ol & - & - & - & 0.25 \\
\hline 122 & 30.62 & $\begin{array}{c}\text { Cholestane-3,5,6-triol, (3beta, 5alpha,6beta)- } \\
\text { Total }\end{array}$ & - & - & - & $\begin{array}{c}0.82 \\
94.32\end{array}$ \\
\hline
\end{tabular}

$\mathrm{RT}^{\mathrm{a}}$ : retention time $(\mathrm{min}) ;$ Peak Area ${ }^{\mathrm{b}}$ : the average of the percentage of peak area relative to the total peak area $(n=3) ;$; - ": not detected.

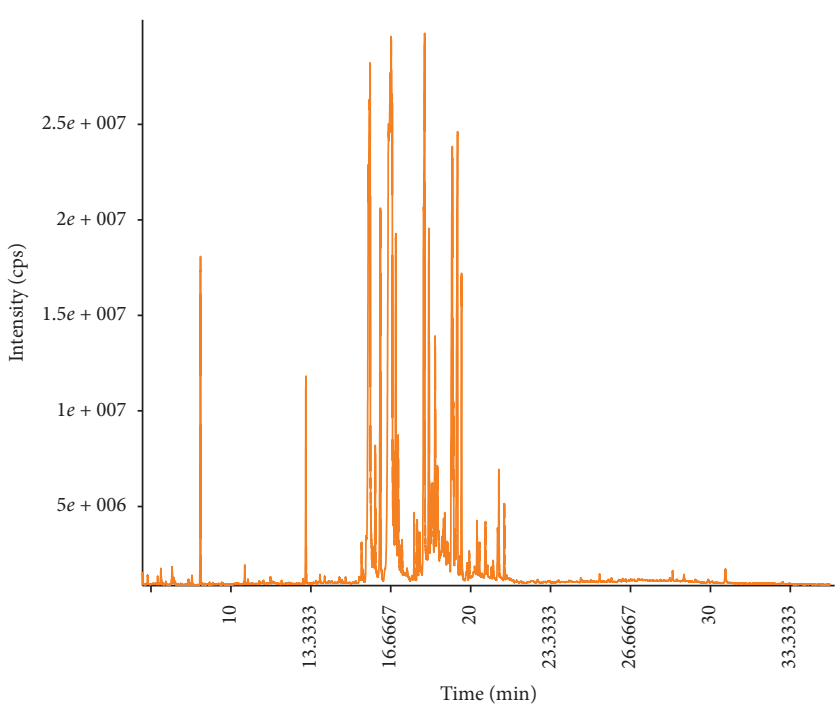

(a)

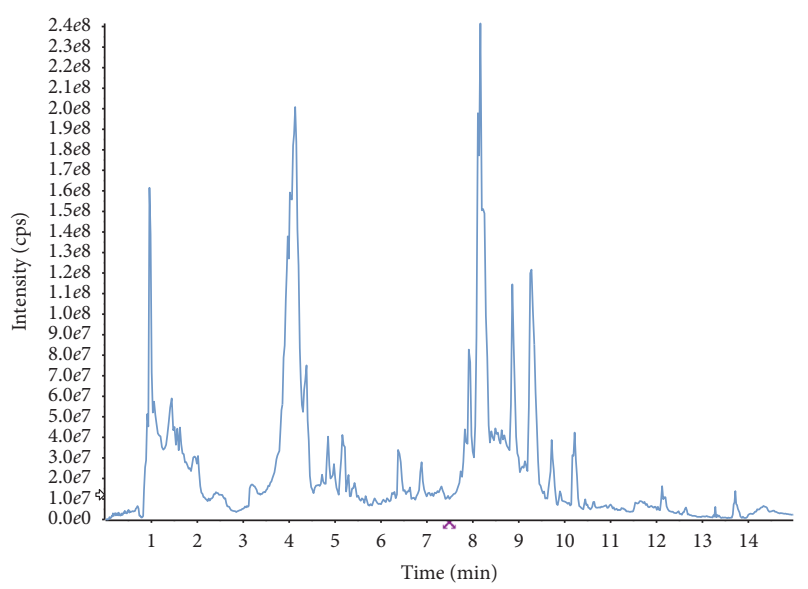

(c)

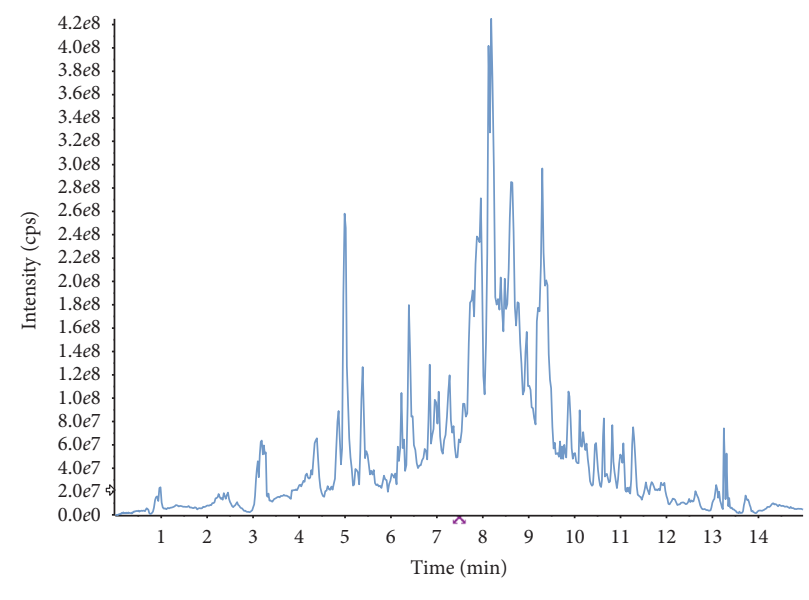

(b)

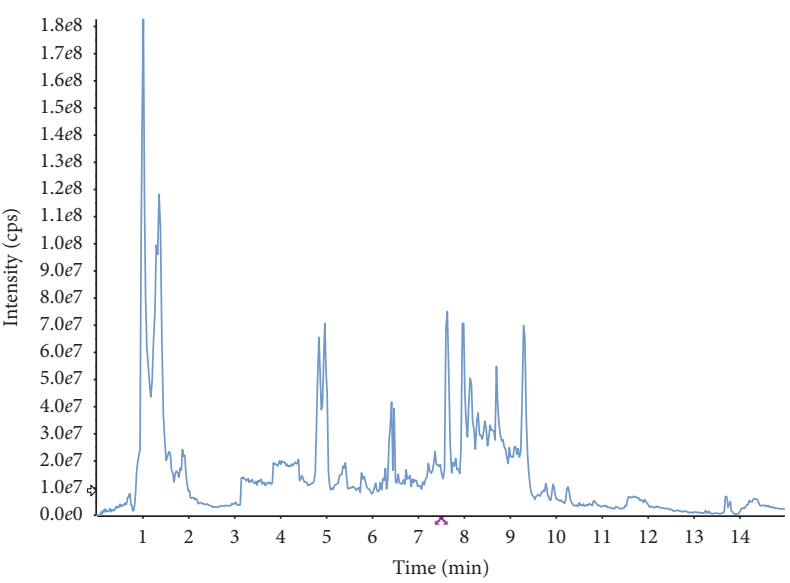

(d)

FIgURe 2: Total ion chromatograms of fractions of celery seeds. (a) PP, (b) PE, (c) PB, and (d) PW.

chemical constituents were adenosine 14.99\%, 15-methylpalmitate $7.18 \%$, ambolin $6.72 \%$, 2-hydroxymuconate semialdehyde $5.21 \%$, and chalconaringenin $4.87 \%$. 58 chemical compositions were identified from PW, mainly sugars, glycosides, acids, and salts, accounting for $84.35 \%$ of the total peak area. Nipecotic acid $11.99 \%$, 3-methyl 1phenyl-3-pentanol $8.05 \%$, mesylate $7.13 \%$, and luteolin 7 galactoside $6.59 \%$ were the main chemical constituents.

Aesculetin, 2-phenylacetamide, 1H-indole-3-carboxaldehyde, alpha-terpinene, and erucamide all existed in PP,
$\mathrm{PE}, \mathrm{PB}$, and $\mathrm{PW}$, and aesculetin accounted for $13.28 \%$, $4.32 \%, 0.56 \%$, and $0.11 \%$. PB, and PW had 19 components in common, including 2-phenylacetamide, 2-acetyl-1-ethylpyrrole, apiin, 2-hydroxymuconate semialdehyde, and 15methylpalmitate. Apigenin was found in both PP and PE, accounting for $11.28 \%$ and $8.48 \%$. It was suggested that the PW, PB, PE and PP exhibited different degree in antiliver fibrosis, which might be related to the active compounds contents combined with the pharmacodynamic experiments and the analysis of components. 
TABLE 6: Effects of monomeric compounds on the proliferation of HSC-LX2 cells (mean \pm SD, $n=3$ ).

\begin{tabular}{|c|c|c|c|}
\hline Group & Dose $\left(\mu \mathrm{g} \cdot \mathrm{mL}^{-1}\right)$ & OD & Inhibition ratio (\%) \\
\hline Solvent control & - & $0.164 \pm 0.012$ & - \\
\hline Blank control & 0 & $1.210 \pm 0.070$ & - \\
\hline Model group(TGF- $\beta 1)$ & 0.1 & $3.325 \pm 0.138^{\# \#}$ & - \\
\hline BJRG & 60 & $0.939 \pm 0.104^{* *}$ & $75.46 \pm 2.11$ \\
\hline Apigenin & $\begin{array}{l}15 \\
30 \\
60 \\
\end{array}$ & $\begin{array}{c}2.746 \pm 0.167^{* \#} \\
1.816 \pm 0.046^{* * \#} \\
1.071 \pm 0.107^{* *} \\
\end{array}$ & $\begin{array}{l}18.33 \pm 1.48 \\
47.72 \pm 1.93 \\
71.32 \pm 2.41 \\
\end{array}$ \\
\hline 5-Methoxypsoralen & $\begin{array}{l}15 \\
30 \\
60 \\
\end{array}$ & $\begin{array}{l}3.337 \pm 0.128 \\
3.382 \pm 0.130 \\
3.454 \pm 0.117 \\
\end{array}$ & $\begin{array}{l}- \\
- \\
-\end{array}$ \\
\hline Apiin & $\begin{array}{l}15 \\
30 \\
60 \\
\end{array}$ & $\begin{array}{l}3.238 \pm 0.177^{\# \#} \\
2.716 \pm 0.184^{* \#} \\
2.349 \pm 0.329^{* \#}\end{array}$ & $\begin{array}{c}2.73 \pm 0.06 \\
19.26 \pm 1.30 \\
30.87 \pm 1.85\end{array}$ \\
\hline Rutin & $\begin{array}{l}15 \\
30 \\
60 \\
\end{array}$ & $\begin{array}{c}3.412 \pm 0.193 \\
3.201 \pm 0.104^{\# \#} \\
2.825 \pm 0.184^{* \# \#}\end{array}$ & $\begin{array}{c}- \\
3.92 \pm 0.57 \\
15.81 \pm 2.68 \\
\end{array}$ \\
\hline Kaempferol & $\begin{array}{l}15 \\
30 \\
60 \\
\end{array}$ & $\begin{array}{c}3.462 \pm 0.161 \\
3.186 \pm 0.252^{\# \#} \\
2.629 \pm 0.177^{* \#}\end{array}$ & $\begin{array}{c}- \\
4.38 \pm 1.02 \\
22.02 \pm 3.93\end{array}$ \\
\hline Luteolin & $\begin{array}{l}15 \\
30 \\
60 \\
\end{array}$ & $\begin{array}{l}3.329 \pm 0.202 \\
3.341 \pm 0.175 \\
3.420 \pm 0.330\end{array}$ & $\begin{array}{l}- \\
- \\
-\end{array}$ \\
\hline Quercetin & $\begin{array}{l}15 \\
30 \\
60\end{array}$ & $\begin{array}{c}3.186 \pm 0.268^{\# \#} \\
2.337 \pm 0.224^{* \#} \\
1.798 \pm 0.218^{* * \#}\end{array}$ & $\begin{array}{c}4.39 \pm 0.75 \\
31.26 \pm 2.11 \\
48.28 \pm 2.74\end{array}$ \\
\hline Mollugin & $\begin{array}{l}15 \\
30 \\
60 \\
\end{array}$ & $\begin{array}{l}3.348 \pm 0.152 \\
3.350 \pm 0.183 \\
3.425 \pm 0.159 \\
\end{array}$ & $\begin{array}{l}- \\
- \\
-\end{array}$ \\
\hline Butylphthalide & $\begin{array}{l}15 \\
30 \\
60 \\
\end{array}$ & $\begin{array}{l}3.029 \pm 0.249^{\# \#} \\
2.366 \pm 0.048^{* \#} \\
1.367 \pm 0.164^{* *}\end{array}$ & $\begin{array}{c}9.36 \pm 1.01 \\
30.33 \pm 2.77 \\
61.93 \pm 3.51\end{array}$ \\
\hline Aesculetin & $\begin{array}{l}15 \\
30 \\
60\end{array}$ & $\begin{array}{c}2.426 \pm 0.245^{* \#} \\
1.979 \pm 0.122^{* * \#} \\
1.277 \pm 0.273^{* *}\end{array}$ & $\begin{array}{l}28.44 \pm 4.01 \\
42.55 \pm 3.14 \\
64.90 \pm 4.21\end{array}$ \\
\hline
\end{tabular}

Compared with the blank control group $\left({ }^{\#} P<0.05,{ }^{\#} P<0.01\right)$; compared with the model group $\left({ }^{*} P<0.05,{ }^{* *} P<0.01\right)$.

3.7. Potential Antiliver Fibrosis Activity of Monomeric Compounds. All apigenin, aesculetin, butylphthalide, quercetin, apiin, kaempferol, and rutin had inhibition effects on HSC-LX2 cell proliferation, and the inhibition effect enhanced with increased compound concentrations (Table 6). Their inhibition rates of high-dose were $71.32 \%$, $64.90 \%, 61.93 \%, 48.28 \%, 30.87 \%, 22.02 \%$, and $15.81 \%$, respectively. 5-methoxypsoralen, luteolin, and mollugin did not have inhibition effects on the proliferation of HSCLX 2 cells. The above results confirmed the antiliver fibrosis compositions of celery seeds and clarified that differences of antiliver fibrosis effects among PW, PB, PE, and PP.

\section{Discussion}

Liver fibrosis is a critical link in the development of cirrhosis or hepatocellular carcinoma, and there is presently no effective treatment for liver fibrosis. Consequently, it is indispensable to develop new drugs to ameliorate liver fibrosis
[34]. In the preceding work, it presented that the alcohol extract of celery seeds could alleviate liver fibrosis. The outcomes of the current work were in keeping with those of earlier studies and further revealed that apigenin, aesculetin, and butylphthalide might have major contribution to the overall antiliver fibrosis activity of celery seeds. In short, it implied that celery seeds might possess potential treatment effect in liver fibrosis.

Transforming growth factor- $\beta 1$ (TGF- $\beta 1$ ) was considered a vital role in the process of liver fibrosis, which could affect hepatic stellate cell (HSC) activation and collagen deposition [35-38]. Therefore, TGF- $\beta 1$ is widely employed to establish a liver fibrosis model in vitro. The activated HSCs lead to hepatic fibrogenesis by excessive production of extracellular matrix (ECM) components [39], such as hyaluronic acid (HA), laminin (LN), and type III procollagen (PCIII) [40-42]. Liver fibrosis is associated with major alterations in both quantity and composition of ECM [43]. In advanced stage, fibrotic liver contains three to ten times 
more ECM than the normal liver [44]. Estimations of serum HA, LN, and PCIII have good prognostic value for liver fibrosis complications $[45,46]$. Compound Biejiaruangan Troche (BJRG) was an SFDA-approved antifibrotic medicine, which was selected as the positive control in this work [47]. It could achieve the effect of preventing and treating liver fibrosis in multiple links and targets. In this work, $60 \%$ ethanol extract of celery seeds (60-extract) showed higher inhibitory rate of HSC-LX2 cells than that of aqueous extract and $95 \%$ ethanol extract. The results of follow-up research manifested that inhibitory effects of petroleum ether (PP) and ethyl acetate (PE) fractions of 60-extract on the proliferation of HSC-LX2 cells stronger than that of n-butyl alcohol (PB) and water-soluble (PW) fractions. Moreover, PP could significantly decrease the contents of HA, LN, and PCIII, which were closer to the positive control. Additionally, PP showed a remarkable proapoptotic effect of HSC-LX2 cells compared with PW, PB, and PE. It concluded that PP could effectively inhibit proliferation and promote apoptosis of HSC-LX2 cells, thereby inhibiting the ECM deposition. Mechanistically, screening of antiliver fibrosis activity of monomeric compounds exhibited that the inhibition rates at high dose of apigenin, aesculetin, butylphthalide, quercetin, apiin, kaempferol, and rutin were $71.32 \%$, $64.90 \%, 61.93 \%, 48.28 \%, 30.87 \%, 22.02 \%$, and $15.81 \%$, respectively. It certified that apigenin, aesculetin, and butylphthalide have major contribution to the antiliver fibrosis activity of celery seeds.

The powerful antifibrotic activity of fractions in celery seeds may be explained by its richness in apigenin, aesculetin, and butylphthalide. This result was consistent with the study by Lee et al. [48], which proved that ethanol-induced cytotoxicity in HepG2 cells and mice were obviously prevented after treatment with aesculetin by the upregulation of antioxidant defense enzymes in the Nrf2/ARE pathway of hepatocytes. Anuradha et al. [49] confirmed the ability of aesculetin to attenuate hepatic fibrosis in NAFLD and its effect on FoxO1 activity. Wang et al. [50] explained that apigenin might exert a protective effect on alcohol-induced liver injury, which might be related to the regulations of hepatic CYP2E1-mediated oxidative stress and PPAR $\alpha$ mediated lipogenic gene expression. It was also discovered that hepatic steatosis and inflammation were ameliorated with treatment of apigenin via regulation of the XO/NLRP3 pathways [51]. Additionally, butylphthalide has been reported in the treatment of cerebrovascular disease. Qiu et al. [52] proved that activation of microglia was prevented; meanwhile, dopaminergic neurons in the substantia nigra were preserved by butylphthalide. Nevertheless, this work first found that butylphthalide has antiliver fibrosis activity in vitro, which provides a theoretical basis for further research on it.

In summary, this work comprehensively and systematically identified the active constituents of celery seeds by GC-TOF-MS and UHPLC-MS/MS and enriched the chemical constituents of celery seeds at this stage. It illustrated that antiliver fibrosis activity clearly elevated with these increased active composition contents. On the whole, a reliable and rapid strategy was successfully established for screening of the antiliver fibrosis activity and active ingredients in celery seeds. PP was verified to be the active fraction of celery seeds with the most powerful antiliver fibrosis effect, and apigenin, aesculetin, and butylphthalide might well be the potential ingredients of antiliver fibrosis activity in celery seeds. To sum up, it is of great significance to elucidate the material basis and pharmacodynamic components of Huganbuzure Granule, Ganbaokang Granule, Fufangzupa Syrup, and Mawuliwusuli Granule of Chinese compound preparations. In addition, apigenin, aesculetin, and butylphthalide may hopefully become the natural products of antiliver fibrosis, which laid a foundation for the subsequent development of celery seeds as antiliver fibrosis drugs.

\section{Conclusions}

According to the biological and chemical analysis of celery seeds, the petroleum ether fraction was verified to be the active fraction of celery seeds with the most powerful antiliver fibrosis effect, and apigenin, aesculetin, and butylphthalide might well be the potential ingredients of antiliver fibrosis activity in celery seeds.

\section{Data Availability}

The data used to support the findings of this study are available from the authors.

\section{Conflicts of Interest}

The authors declare that there are no conflicts of interest regarding the publication of this article.

\section{Authors' Contributions}

Junping $\mathrm{Hu}$ acquired funding for the research and designed the work; Ming Qiao and Jianhua Yang wrote the manuscript; and Ming Qiao, Yi Zhu, Jianhua Yang, Yao Zhao, Xiaomei Wang, and Xinling Wang performed the experiment and analysed the data. All authors discussed the results and approved the final manuscript. Ming Qiao and Jianhua Yang contributed equally to this work.

\section{Acknowledgments}

This research was supported by the National Natural Science Foundation of China (no. 81560688).

\section{References}

[1] P. Melgar-Lesmes, M. Perramon, and W. Jimenez, "Roles of the hepatic endocannabinoid and apelin systems in the pathogenesis of liver fibrosis," Cells, vol. 8, no. 11, pp. 265-276, 2019.

[2] H. Ebrahimi, M. Naderian, and A. A. Sohrabpour, "New concepts on pathogenesis and diagnosis of liver fibrosis; a review article," Middle East Journal of Digestive Diseases, vol. 8, no. 3, pp. 166-178, 2016.

[3] G. Li, Y. Zhou, D. M.-Y. Sze et al., "Active ingredients and action mechanisms of Yi Guan Jian decoction in chronic 
hepatitis B patients with liver fibrosis," Evidence-Based Complementary and Alternative Medicine, vol. 2019, Article ID 2408126, 13 pages, 2019.

[4] Y. Zhang, J. Liu, Y. Ma et al., "Integration of highthroughput data of microRNA and mRNA expression profiles reveals novel insights into the mechanism of liver fibrosis," Molecular Medicine Reports, vol. 19, no. 1, pp. 115-124, 2019.

[5] Y. Zhou, X. Lv, H. Qu et al., "Differential expression of circular RNAs in hepatic tissue in a model of liver fibrosis and functional analysis of their target genes," Hepatology Research, vol. 49, no. 3, pp. 324-334, 2019.

[6] D. Yang, "Research progress of receptor targeted hepatic stellate cell in treatment of liver fibrosis," Zhonghua Gan Zang Bing Za Zhi, vol. 26, no. 8, pp. 630-632, 2018.

[7] S. Tan, Y. Lu, M. Xu et al., " $\beta$-arrestin1 enhances liver fibrosis through autophagy-mediated snail signaling," The FASEB Journal, vol. 33, no. 2, pp. 2000-2016, 2019.

[8] Z. Liu, P. Zhu, L. Zhang et al., "Autophagy inhibition attenuates the induction of anti-inflammatory effect of catalpol in liver fibrosis," Biomedicine \& Pharmacotherapy, vol. 103, no. 16, pp. 1262-1271, 2018.

[9] J.-B. Qiao, Q.-Q. Fan, L. Xing et al., "Vitamin A-decorated biocompatible micelles for chemogene therapy of liver fibrosis," Journal of Controlled Release, vol. 283, no. 21, pp. 113-125, 2018.

[10] W. Kooti and N. Daraei, "A review of the antioxidant activity of celery (Apium graveolens L)," Journal of Evidence-Based Complementary \& Alternative Medicine, vol. 22, no. 4, pp. 1029-1034, 2017.

[11] P. Chonpatho, P. Boonruam, W. Sukketsiri, P. Hutamekalin, and M. Sroyraya, "The antioxidant and neurochemical activity of Apium graveolens L. and its ameliorative effect on MPTPinduced Parkinson-like symptoms in mice," BMC Complementary and Alternative Medicine, vol. 18, no. 1, pp. 169-182, 2018.

[12] N. Choosri, S. Tanasawet, P. Chonpathompikunlert, and W. Sukketsiri, "Apium graveolens extract attenuates adjuvant induced arthritis by reducing oxidative stress," Journal of Food Biochemistry, vol. 41, no. 1, pp. 12276-12283, 2017.

[13] K. Dolati, H. Rakhshandeh, M. Golestani, F. Forouzanfar, R. Sadeghnia, and H. R. Sadeghnia, "Inhibitory effects of Apium graveolens on xanthine oxidase activity and serum uric acid levels in hyperuricemic mice," Preventive Nutrition and Food Science, vol. 23, no. 2, pp. 127-133, 2018.

[14] Y. He, Y. Shi, A. Zhang, X. Zhang, J. Sun, and L. Tian, "Lipidlowering and antioxidative effects of Apium graveolens L. root flavonoid extracts," RSC Advances, vol. 9, no. 46, pp. 26757-26767, 2019.

[15] D. Chen, L. D. Melton, D. J. Gillivray, T. M. Ryan, and P. J. Harris, "Changes in the orientations of cellulose microfibrils during the development of collenchyma cell walls of celery (Apium graveolens L.)," Planta, vol. 250, no. 6, pp. 1819-1832, 2019.

[16] P. Li, J. Jia, D. Zhang, J. Xie, X. Xu, and D. Wei, "In vitro and in vivo antioxidant activities of a flavonoid isolated from celery (Apium graveolens L.)," Food and Function, vol. 5, no. 1, pp. 50-60, 2014.

[17] Z. Uddin, A. A. Shad, and J. Bakht, "In vitro antimicrobial, antioxidant activity and phytochemical screening of Apium graveolens," Pakistan Journal of Pharmaceutical Sciences, vol. 28, no. 3, pp. 1699-1704, 2015.

[18] D. Iyer and U. K. Patil, "Assessment of antihyperlipidemic and antitumor effect of isolated active phytoconstituents from Apium graveolens L. through bioassay-guided procedures,"
Journal of Dietary Supplements, vol. 16, no. 2, pp. 193-206, 2019.

[19] J.-X. Liu, K. Feng, G.-L. Wang, Z.-S. Xu, F. Wang, and A.-S. Xiong, "Elevated $\mathrm{CO}_{2}$ induces alteration in lignin accumulation in celery (Apium graveolens L.)," Plant Physiology and Biochemistry, vol. 127, no. 6, pp. 310-319, 2018.

[20] K. Feng, J.-X. Liu, A.-Q. Duan et al., "AgMYB2 transcription factor is involved in the regulation of anthocyanin biosynthesis in purple celery (Apium graveolens L.)," Planta, vol. 248, no. 5, pp. 1249-1261, 2018.

[21] D. Chen, L. Melton, Z. Zujovic, and P. J. Harris, "Developmental changes in collenchyma cell-wall polysaccharides in celery (Apium graveolens L.) petioles," BMC Plant Biology, vol. 19, no. 1, pp. 81-103, 2019.

[22] Y. Yusni, H. Zufry, F. Meutia, and K. Sucipto, "The effects of celery leaf (Apium graveolens L.) treatment on blood glucose and insulin levels in elderly pre-diabetics," Saudi Medical Journal, vol. 39, no. 2, pp. 154-160, 2018.

[23] M.-Y. Li, J.-X. Liu, J.-N. Hao et al., "Genomic identification of AP2/ERF transcription factors and functional characterization of two cold resistance-related AP2/ERF genes in celery (Apium graveolens L.)," Planta, vol. 250, no. 4, pp. 1265-1280, 2019.

[24] Y. A. Crespo, S. L. Bravo, Y. G. Quintana, A. S. T. Cabrera, A. B. del Sol, and D. M. Guzmán Mayancha, "Evaluation of the synergistic effects of antioxidant activity on mixtures of the essential oil from Apium graveolens $\mathrm{L}$., Thymus vulgaris $\mathrm{L}$. and Coriandrum sativum L. using simplex-lattice design," Heliyon, vol. 5, no. 6, pp. 1942-1966, 2019.

[25] H. B. Huang, L. Jiang, and J. Liu, "Study on the chemical constituents of Glechoma longituba," Journal of Chinese Medicinal Materials, vol. 40, no. 4, pp. 844-847, 2017.

[26] X. Luo, X. J. Wang, and Y. W. Zhao, "Chemical constituents from Notopterygium incisum," Chinese Traditional and Herbal Drugs, vol. 47, no. 9, pp. 1492-1495, 2016.

[27] Y. P. Lin, Y. L. Zong, and H. He, "Chemical constituents of the aerial parts of Seseliyunnanense Franch," Natural Products Research Development, vol. 19, no. 836, pp. 798-800, 2007.

[28] X. J. Wang, X. Luo, and J. M. Zhou, "Chemical constituents from rhizomes of Rhodiola wallichiana var. cholaensis and their protective effects on myocardium," Chinese Traditional and Herbal Drugs, vol. 47, no. 16, pp. 2822-2826, 2016.

[29] M. M. Yan, S. J. Xiao, and F. Chen, "Chemical constituents of Hypericum ascyron L.," Natural Products Research Development, vol. 26, no. 11, pp. 1785-1788, 2014.

[30] G. Ablikim, N. Yadikar, and H. Aisa, "Chemical constituents from the residue of Lavandula angustifolia and their biological activities," Chinese Traditional Patent Medicine, vol. 41, no. 4, pp. 818-822, 2019.

[31] R. L. Li, P. L. Wu, and C. R. Li, "Study on the chemical constituents of the branches and leaves of Juniperus formosana," West China Journal of Pharmaceutical Sciences, vol. 34, no. 1, pp. 5-9, 2019.

[32] Z. Wang, S. M. Zhao, and G. Z. Zeng, "Chemical constituents from roots and rhizomes of Rubia oncotricha and their cytotoxic activities," China Journal of Chinese Materia Medica, vol. 43, no. 22, pp. 4462-4468, 2018.

[33] W. H. Chen, G. Shen, and H. S. Chen, "High purity preparation and identification of three phthlide compounds from Apium graveolens," Journal of Pharmaceutical Practice, vol. 35, no. 2, pp. 138-140, 2017.

[34] M. M. Salah, A. A. Ashour, T. M. Abdelghany, A.-A. H. Abdel-Aziz, and S. A. Salama, "Pirfenidone alleviates 
concanavalin A-induced liver fibrosis in mice," Life Sciences, vol. 6, no. 12, pp. 1169-1182, 2019.

[35] D. Huang, T. Lin, S. Wang et al., "The liver fibrosis index is superior to the APRI and FIB-4 for predicting liver fibrosis in chronic hepatitis B patients in China," BMC Infectious Diseases, vol. 19, no. 1, pp. 878-891, 2019.

[36] J. Day, P. Patel, J. Parkes, and W. Rosenberg, "Derivation and performance of standardized enhanced liver fibrosis (ELF) test thresholds for the detection and prognosis of liver fibrosis," The Journal of Applied Laboratory Medicine, vol. 3, no. 5, pp. 815-826, 2019.

[37] H.-C. Tsay, Q. Yuan, A. Balakrishnan et al., "Hepatocytespecific suppression of microRNA-221-3p mitigates liver fibrosis," Journal of Hepatology, vol. 70, no. 4, pp. 722-734, 2019.

[38] F. Zhang, M. Hao, H. Jin et al., "Canonical hedgehog signalling regulates hepatic stellate cell-mediated angiogenesis in liver fibrosis," British Journal of Pharmacology, vol. 174, no. 5, pp. 409-423, 2017.

[39] F. Yu, B. Chen, P. Dong, and J. Zheng, "Hotair epigenetically modulates PTEN expression via MicroRNA-29b: a novel mechanism in regulation of liver fibrosis," Molecular Therapy, vol. 25, no. 1, pp. 205-217, 2017.

[40] P. Xiong, J. Zhang, D. Xu et al., "Positive feedback loop of YB1 interacting with Smad2 promotes liver fibrosis," Biochemical and Biophysical Research Communications, vol. 484, no. 4, pp. 753-761, 2017.

[41] T. Higashi, S. L. Friedman, and Y. Hoshida, "Hepatic stellate cells as key target in liver fibrosis," Advanced Drug Delivery Reviews, vol. 121, no. 12, pp. 27-42, 2017.

[42] W. Wang, R. Dong, Y. Guo et al., "CircMTO1 inhibits liver fibrosis via regulation of miR-17-5p and Smad7," Journal of Cellular and Molecular Medicine, vol. 23, no. 8, pp. 54865496, 2019.

[43] F. Alegre, P. Pelegrin, and A. Feldstein, "Inflammasomes in liver fibrosis," Seminars in Liver Disease, vol. 37, no. 2, pp. 119-127, 2017.

[44] X. K. Zhao, L. Yu, and M. L. Cheng, "Focal adhesion kinase regulates hepatic stellate cell activation and liver fibrosis," Scientific Reports, vol. 7, no. 1, pp. 4032-4063, 2017.

[45] Z. Dong, L. Su, S. Esmaili et al., "Adiponectin attenuates liver fibrosis by inducing nitric oxide production of hepatic stellate cells," Journal of Molecular Medicine, vol. 93, no. 12, pp. 1327-1339, 2015.

[46] L. Chen, R. Chen, S. Kemper, M. Cong, H. You, and D. R. Brigstock, "Therapeutic effects of serum extracellular vesicles in liver fibrosis," Journal of Extracellular Vesicles, vol. 7, no. 1, pp. 1461-1505, 2018.

[47] J. Qu, Z. Yu, Q. Li et al., "Blocking and reversing hepatic fibrosis in patients with chronic hepatitis B treated by traditional Chinese medicine (tablets of biejia ruangan or RGT): study protocol for a randomized controlled trial," Trials, vol. 15, no. 1, pp. 438-445, 2014.

[48] J. Lee, J. Yang, J. Jeon, H. Sang Jeong, J. Lee, and J. Sung, "Hepatoprotective effect of aesculetin on ethanol-induced liver injury in human HepG2 cells and C57BL/6J mice," Journal of Functional Foods, vol. 40, no. 18, pp. 536-543, 2018.

[49] A. Pandey, P. Raj, S. K. Goru et al., "Aesculetin ameliorates hepatic fibrosis in high fat diet induced non-alcoholic fatty liver disease by regulation of FoxO1 mediated pathway," Pharmacological Reports, vol. 69, no. 4, pp. 666-672, 2017.

[50] F. Wang, J.-C. Liu, R.-J. Zhou et al., “Apigenin protects against alcohol-induced liver injury in mice by regulating hepatic CYP2E1-mediated oxidative stress and $\operatorname{PPAR} \alpha$-mediated lipogenic gene expression," Chemico-Biological Interactions, vol. 275, no. 14, pp. 171-177, 2017.

[51] Y. Lv, X. Gao, Y. Luo et al., "Apigenin ameliorates HFDinduced NAFLD through regulation of the XO/NLRP3 pathways," The Journal of Nutritional Biochemistry, vol. 71, no. 8, pp. 110-121, 2019.

[52] H. Qiu, J. Ma, H. Wu, and C. Ding, "DL-3-n-butylphthalide improves ventricular function, and prevents ventricular remodeling and arrhythmias in post-MI rats," NaunynSchmiedeberg's Archives of Pharmacology, vol. 391, no. 6, pp. 627-637, 2018. 